\title{
KEY ISSUES: A ROUND TABLE DISCUSSION
}

\author{
T. M. BROWN ${ }^{1}$, P. DEMARQUE 2 , R. NOYES ${ }^{3}$, F. PRADERIE ${ }^{4}$, I. W. \\ ROXBURGH ${ }^{5}$, E. SCHATZMAN ${ }^{6}$ (CHAIR) \\ ${ }^{1}$ High Altitude Observatory, National Center of Atmospheric Research, \\ P. O. Box 3000, Boulder, CO 80307, USA \\ ${ }^{2}$ Yale University, Department of Astronomy, P.(). Box 6606, New Haven, \\ CT 06511, USA \\ ${ }^{3}$ Smithonian Astrophysical Observatory, 60 Garden Street, Cambridge, \\ MA 02138, USA \\ 4 IJESPA, URA CNRS 264, Observatoire de Paris, 92195 Meudon Cedex, \\ France \\ ${ }^{5}$ School of Mathematical Science, Queen Mary College, Mile End Road, \\ London, E14NS, UK ${ }^{6}$ DASGAI, URA CNRS 335, Observatoire de Paris, \\ 92195 Meudon Cedex, France
}

\section{INTRODUCTION by Evry Schatzman}

We have taken part to an exceptionally rich colloquium, characterized by a large amount of information in all fields of physics, and a remarkable collection of observational data. Our understanding of what is going on inside the stars has changed in a radical manner during the last years, on one side because the observations are bringing new kinds of information, and on the other side because theory in its development is taking into account a number of processes, some of them having been completely ignored only ten years ago.

It is impossible to draw here a complete list of problems. There are many cases where some inconsistency could be found in the theory itself or some contradiction between observational data and theory. I recommended that such a list should be drawn carefully. The participants to the round table discussion will give their contribution, helping to open the way to new fields of research and to new discoveries.

In the following. you will find the conclusions of Pierre Demarque, Ian Roxburgh, Bob Noyes, Timothy Brown, Françoise Praderie and myself.

\section{INTERNAL DYNAMICS OF STARS by Pierre Demarque}

I want to say a few words about the internal dynamics of stars, which has been a topic of growing interest in the last few years. This interest is likely to continue to grow in the next decade and we can expect dramatic advances in the areas of convection, rotation and non-radial pulsation.

Numerical models of stellar convection will become increasingly realistic. For example, the study of spectral line bisectors will lead to a better understanding of the outer convective layers of cool stars. For these stars, models of surface convection zones free of the arbitrary mixing length parameter and 
based on sounder fluid dynamics will become available, yielding an improved envelope structure and (at last!) permitting the calculation of stellar radii from first principles. A better understanding of penetrative convection and overmixing (convective overshoot) will follow. The role of magnetic fields in convective patterns and transport will also be increasingly studied.

Thanks to the recent observations of rotational surface velocities, of spectroscopic abundances of the light elements $\mathrm{Li}, \mathrm{Be}$ and $\mathrm{B}$, and of the products of CNO processing mixed to stellar surfaces, progress has already been made in exploring the processes of angular momentum transfer and redistribution in stars. The light elements $\mathrm{Li}$ and Be probe the rotational history of stellar envelopes, while the $\mathrm{CNO}$ isotopes, which are processed by nuclear reactions in the deep interior probe the degree of mixing from the core to the surface. More such abundance observations, particularly in star clusters, young and old, metal-rich and metal-poor, will constrain the theory. Together with the rotational velocities, which reveal the characteristic timescales of the surface braking due to the stellar winds, they provide in the interior stringent tests of angular momentum transfer mechanisms and of their mixing efficiency. Abundance spreads would also be particularly significant.

Finally, there is the exciting prospect of using seismic techniques to probe stellar internal dynamics. In this context, the PRISMA space mission, proposed to ESA and featured at this colloquium, is very promising. My main comment here is that, like all techniques in astrophysics, seismology will yield best scientific results only if it is done in parallel with detailed observations of chemical abundances and other basic parameters (such as parallaxes) of the target stars, and with the help of theoretical modeling.

\section{SOME PROBLEMS IN STELLAR STRUCTURE AND EVOLUTION}

\section{by Ian W. Roxburgh}

\section{Convection}

The first point I wish to make is that convection is a global phenomenon. Since the convective "mixing time" is very much smaller than both the radiative and evolutionary time scales, a convective zone should be considered as a global system; what happens at one part of the zone effects what happens at another and the whole zone achieves some sort of global (statistical) equilibrium. Local theories, such as the mixing length model, cannot capture this essential feature of convection and some different approach is needed.

Numerical simulations (cf Stein and Nordlund 1989) indicate that "convective plumes" extend over most of the convective region and penetrate into the surrounding stable layers. "Theories" incorporating such plumes need to be developed and applied to models of convective zones, and perhaps more importantly to convective cores. Such high velocity plumes may be of considerable importance in the mixing of chemical elements beyond the boundary of convectively unstable regions, since the cumulative effect of deep penetration by plumes occupying only a small fraction of the volume could produce effective mixing of chemical elements in the evolutionary time scale of a star.

A model of convection incorporating downward moving plumes was developed in the early 1960's by Hoyle (cf Faulkner et al 1965) who observed that 
downward moving material becomes increasingly thermally isolated from its surroundings and may penetrate all the way from the top to the bottom of the layer; this model was modified by Roxburgh and Vickers (see Vickers 1970) who incorporated the change in the area occupied by such plumes as a function of depth, but this "theory" has been little used since that time. A "plume" model was also developed by Schmidt et al (1984), [see also Zahn 1991] but again this has not been developed to the stage where it can be used in modelling stellar convective regions. Upward moving plumes may also be important; in stellar interiors the decrease of the "thermal conduction" $\left(4 \mathrm{acT} \mathrm{T}^{3} / 3 \kappa \rho\right)$ with decreasing temperature implies that it is the upward moving elements that become increasingly thermally isolated from the surrounding regions rather than the downward moving elements.

Convective overshooting - the integral constraint

Next I wish to comment on the so called "Roxburgh criterion" for convective overshooting which follows from integrating the entropy equation over the volume of the convective zone (Roxburgh 1976, 1978, 1989)

$$
\int_{0}^{\mathrm{r}_{c}}\left(\mathrm{~L}_{\mathrm{rad}}-\mathrm{L}_{\mathrm{nuc}}\right) \frac{1}{\mathrm{~T}^{2}} \frac{\mathrm{dT}}{\mathrm{dr}} \mathrm{dr}=\int_{0}^{\mathrm{r}_{\mathrm{c}}} \frac{\Phi}{\mathrm{T}} 4 \pi \mathrm{r}^{2} \mathrm{dr}>0
$$

To obtain this result turbulent averages have been replaced by functions of the mean field; recent numerical calculations, admittedly in a parameter regime removed from that applicable to stellar interiors, suggests that this approximation is reasonable (Roxburgh and Simmons 1992). I should however emphasise that the integral condition was derived to give an estimate of the uncertainty in standard stellar models (where the boundary of convective cores is determined by the condition $\mathrm{L}_{\mathrm{rad}}=\mathrm{L}_{\mathrm{nuc}}$ ), not to provide a "correct" determination of the extent of convective penetration. Since the viscous dissipation $\Phi$ is positive definite the condition with $\Phi=0$ provides a useful estimate of the upper limit on the extent of convective overshooting. To illustrate the point consider stars with very small convective cores and expand $\mathrm{L}_{\mathrm{rad}}$ and $\mathrm{L}_{\mathrm{nuc}}$ in powers of $\mathrm{r}$ as

$$
L_{\mathrm{rad}}=\mathrm{A}_{0} \mathrm{r}^{3}-\mathrm{A}_{2} \mathrm{r}^{5}+\ldots, \quad \mathrm{L}_{\mathrm{nuc}}=\mathrm{B}_{0} \mathrm{r}^{3}-\mathrm{B}_{2} \mathrm{r}^{5}+\ldots
$$

The "classical" core boundary where $\mathrm{L}_{\mathrm{rad}}=\mathrm{L}_{\text {nuc }}$ is then at

$r_{0}=\left[\left(A_{0}-B_{0}\right) /\left(A_{2}+B_{2}\right)\right]^{1 / 2}$. Expanding $T=T_{0}-T_{2} r^{2}+T_{4} r^{4}+\ldots\left(T_{2}<0\right)$, and evaluating the integral condition (with $\Phi=0$ ) gives the core boundary at $\mathrm{r}$ $=\mathrm{r}_{c}$ where

$$
\mathrm{r}_{\mathrm{c}}^{2}<\frac{7}{5}\left(\frac{\mathrm{A}_{0}-\mathrm{B}_{0}}{\mathrm{~A}_{2}-\mathrm{B}_{2}}\right)=\frac{7}{5} \mathrm{r}_{0}^{2}
$$

Hence the maximum extension of the core due to convective overshooting is 0.18 ro.

In modelling the extension of convective cores due to overshooting several authors extend the unstable core by some fraction of the local scale height; since the scale height tends to infinity as $r$ tends to zero this can give an overshoot region much larger than the limit from the integral constraint, and give solar models in which a convective core remains up to the present time. In the solar models I have computed with overshooting limited by the integral constraint, 
a ${ }^{3} \mathrm{He}$ and ${ }^{12} \mathrm{C}$ driven convective core lasts considerable longer than in models without overshooting (due to the transport of ${ }^{3} \mathrm{He}$ and ${ }^{12} \mathrm{C}$ into the centre), but nevertheless the core dies away at an age of about $10^{8}$ years.

\section{Mixing - the effect of chemical composition gradients}

Next I wish to stress a point I have made several times before: the existence of instabilities is not sufficient to ensure mixing of chemical elements; mixing requires sufficient energy input to overcome the stabilising effect of a chemical composition gradient (cf Roxburgh 1984, 1985, 1991). This can be demonstrated as follows. Consider a "blob" of fluid raised sufficiently slowly through a distance $\mathbf{z}$ in the vertical direction so that the pressure temperature inside the blob are the same as the surroundings; the density excess $\delta \mathbf{r}$ at $z$, and the total work per unit mass $\mathrm{W}$ done in interchanging two blobs a distance $\mathbf{k}$ apart, are

$$
\frac{\delta \rho}{\rho}=\frac{\delta \mu}{\mu}=-\frac{\mathbf{z} \cdot \nabla \mu}{\mu}, \quad \mathrm{W}=2 \int_{0}^{\mathrm{k}} \mathrm{g}_{\mathrm{z}} \frac{\delta \rho}{\rho}=-(\mathbf{k} \cdot \mathbf{g}) \frac{\mathbf{k} \cdot \nabla \mu}{\mu}
$$

where $\mathbf{g}$ is the acceleration due to gravity. Any process that mixes regions of a star has to provide this energy. If the major part of the star is to be mixed then the distance $\mathbf{k}$ is of order of magnitude the radius of the star the total energy required is of the order $\mathrm{W} \approx \Delta \mu / \mu\left(\mathrm{GM}^{2} / \mathrm{R}\right)$. With $\Delta \mu \approx(d \mu / d t) \mathrm{t}_{0}$, where $t_{0}$ is the age of the star, mixing would require a continuous rate of input of energy per unit mass per unit time of approximately $\Phi(1 / \mu) d \mu / d t$, where $\Phi$ is the absolute value of the gravitational potential. Most energy sources, eg. instabilities driven by differential rotation and meridional circulation, do not satisfy this condition in stellar interiors, although they can provide enough energy in the outer layers. One effective mixing mechanism is convection, the energy source being ultimately the nuclear reactions and the rate of production of energy per unit mass from nuclear sources is considerably in excess of that required to overcome the growing composition gradient.

${ }^{3} \mathrm{He}$ instability in solar type stars

Another outstanding problem is the ${ }^{3} \mathrm{He}$ instability in solar type stars (Dilke and Gough, 1972, Christensen-Dalsgaard et al 1974), in which a non radial gmode is excited by nuclear reactions, the energy input over an oscillation period from the burning of ${ }^{3} \mathrm{He}$ exceeding the energy losses by radiation $\left({ }^{12} \mathrm{C}\right.$ can also drive such an instability). An estimate of the energy available is obtained from calculating the rate of production of excess ${ }^{3} \mathrm{He}$ at the time instability sets in and assuming this to be converted into kinetic energy. This is more than sufficient to overcome the stabilising effect of the composition gradient and at least on energetic grounds this instability is capable of causing mixing.

The consequences of this instability have not been fully explored and it is usual to ignore it when calculating evolutionary models of the sun and stars. This is not reasonable. One possibility is that the instability leads to finite amplitude oscillations where the excess rate of burning of ${ }^{3} \mathrm{He}$ during an oscillation maintains the distribution near the marginally stable state; this requires quite large amplitudes. Resulting models of the present Sun have a higher central hydrogen abundance, lower central temperature and a reduced Solar Neutrino Flux (Roxburgh 1985, 1987). Another possibility is that the oscillation breaks down into mild turbulence which diffuses the excess ${ }^{3} \mathrm{He}$ into the centre where 
it is burnt (Roxburgh 1984, 1991), the diffusion being such as to maintain the star near marginal instability. Evolutionary models of the Sun including this diffusion do not show any major changes over standard solar models. Another possibility (Dziembowski, private communication) is that the unstable mode couples to stable modes and the oscillation has quite small amplitudes with negligible effect on the internal structure and evolution.

The non linear evolution of this instability and its possible effect on solar and stellar evolution needs further investigation.

Magnetic fields in stellar interiors

Magnetic fields may or may not be important in stellar evolution but we cannot simply ignore their prescence we need to quantify their effects. The matter inside stars is essentially a fully ionised plasma and even if by some "fluke" a star had no initial magnetic field, a field would be generated from the turbulent motions and/or differential rotation by the "battery" effect of the electron partial pressure. (cf Biermann 1950, Roxburgh 1966). In the surface layers of the Sun and many other stars, magnetic fields play a dominant role, and much effort has been expended in trying to understand the generation of the solar magnetic field by a dynamo action induced by differential rotation and the helicity of the turbulent motions in the outer convective zone.

But what about stellar interiors? Many stars are thought to have a central convective core during most of their main sequence phase, and even smaller mass stars like the Sun probably had a small convective core in their early main sequence phase. Why should not a dynamo operate in such convective cores? Indeed we might expect a turbulent dynamo to operate in this phase and build up a strong field even in the absence of rotation. Results from studies of homogeneous MHD turbulence (cf Durney, de Young and Passot 1990, Durney, de Young and Roxburgh 1992) indicate that even in the absence of helicity, kinetic energy of the turbulence is converted into magnetic energy, and that this magnetic energy cascades to large scale modes. With significant helicity the magnetic energy can exceed the kinetic energy of the turbulence. What happens to such a field generated in a stellar convective core? The perceived wisdom in the case of the solar convective envelope is that magnetic fields "float" to the top of the outer convective zone due to buoyancy (the density inside a flux tube being less than that of the surroundings), but a stellar convective core is surrounded by a stable region so where does the flux go to? Does it accumulate at the top of the convective core? If so what effect does it have on the structure and dynamics of the boundary region? Does it inhibit convective penetration or enhance mixing by producing a time varying magnetic force. What limits the input of kinetic energy into magnetic energy? Could the field become large enough to suppress the convection? These problems require further study.

Magnetic fields and differential rotation

Another problem that has concerned me for some time is the effect of "phase mixing" in the redistribution of angular momentum in differentially rotating stars and in spin down of stars due to angular momentum loss in a stellar wind (cf Roxburgh 1985,1991). In the absence of magnetic instabilities differential rotation generates a large toroidal field from a small poloidal field, this toroidal field reacts back on the differential rotation and the system undergoes torsional oscillations with a period given by the local Alfvén travel time along the poloidal 
field. If we neglect the effect of any poloidal motions induced by the oscillating field then the problem is governed by the two evolution equations

$$
\begin{aligned}
& \frac{\partial\left(\rho \Omega \mathrm{r}^{2} \sin ^{2} \theta\right)}{\partial \mathrm{t}}=\frac{1}{4 \pi} \mathrm{B}_{\mathrm{p}} \cdot \nabla\left(\mathrm{B}_{\phi} \mathrm{r} \sin \theta\right)+\operatorname{div}\left(\rho \nu \mathrm{r}^{2} \sin ^{2} \theta \cdot \nabla \Omega\right) \\
& \frac{\partial \mathbf{B}_{\phi}}{\partial \mathrm{t}}=\mathrm{r} \sin \theta\left(\mathbf{B}_{\mathrm{p}} \cdot \nabla \Omega\right)+\mathrm{r} \sin \theta \operatorname{div}\left(\frac{\eta}{\mathrm{r}^{2} \sin ^{2} \theta} \nabla\left(\mathrm{r} \sin \mathbf{B}_{\phi}\right)\right)
\end{aligned}
$$

where $\mathbf{B}_{p}(r, \theta)$ is the poloidal field, $\mathbf{B}_{\phi}(r, \theta)$ the toroidal field, $\Omega(r, \theta)$ the angular velocity, $(r, \theta, \phi)$ are spherical polar coordinates $\nu$ the kinematic viscosity and $\eta$ the magnetic diffusivity. With $\eta$ and $\nu$ zero these equations only contain derivatives of $\Omega$ and $\mathbf{B} \phi$ along the poloidal field lines $(\mathbf{B} \phi \cdot \nabla)$ and not perpendicular to them; poloidal field lines are therefore uncoupled and have different oscillation periods; neighbouring field lines therefore rapidly get out of phase. This "phase mixing" creates large gradients in the toroidal field and rotation, thereby causing enhanced dissipation of energy. Since dissipation converts magnetic and kinetic energy into heat, and since the source of energy is the differential rotation, we might expect the system to evolve towards the lowest energy state. But for a given angular momentum the state of lowest energy is that of uniform rotation, so we expect such phase mixing to lead to uniform rotation. Detailed numerical calculations are in progress by Gerado Ocana and myself (cf Roxburgh 1991). Charboneau and MacGregor have also undertaken similar calculations (see these proceedings) although they appear to have replaced $(\mathbf{B} \cdot \nabla \mathbf{B})_{\phi}$ by $\left(\mathbf{B}_{p} \cdot \nabla\right) \mathbf{B}_{\phi}$ rather than $\left[\mathbf{B}_{p} \cdot \nabla\left(\mathbf{r} \sin \theta \mathbf{B}_{\phi}\right)\right] /(\mathbf{r} \sin \theta)$ and to have dropped a term involving the derivative of the density gradient from equation (5). Numerical calculations by Ocana and Roxburgh will be published in the near future.

\section{STUDY OF STELLAR ACTIVITY MECHANISMS USING PRISMA}

\section{by Robert Noyes}

A major focus of PRISMA is study of the nature of stellar magnetic activity. This can be done through detailed comparison of stellar behavior observed by PRISMA with analogous behavior observed on the sun. Although PRISMA is not expected to remain operational over a typical stellar magnetic activity cycle, nevertheless it can tell us much about magnetic activity through repeated observations of total luminosity, magnetic activity indicators, and p-mode frequencies in particular stars.

It is now well known that the solar luminosity varies with the envelope of the activity cycle, being about $0.1 \%$ larger near activity maximum than near activity minimum. Younger stars, such as those of the age of the Hyades (approximately 600 million years), also to show a variation, but with opposite sign relative to their overall magnetic activity level (e.g. Radick et al 1990). This may be because their much larger dark starspots overwhelm the increased brightness due to the associated plages, in contrast to the case for the sun. Another activityrelated phenomenon seen on the sun is a slight shift of p-mode frequencies toward higher frequencies when magnetic activity increases; the frequency-dependence of the shift implies an activity-related perturbation of structure just below the surface (Libbrecht and Woodard 1990). The frequency shifts are closely correlated with individual episodes of surface magnetic activity averaged over the 
sun, rather than the 11-year cycle timescale itself (Woodard et al 1991). We do not know, however, how this interesting behavior manifests itself on other stars. It seems likely that as younger stars undergo their much larger episodes of magnetic activity growth and decay, the p-mode frequencies will be found to follow in step, but with a much larger fractional change than seen in the sun. However the fact that on younger stars the associated luminosity changes are seen to be oppositely-directed from those on the sun suggests that there could be some surprises. In any case, following the p-mode frequency changes to the expected much larger amplitudes in more active stars may provide more information on the nature of stellar activity and its subphotospheric roots than we can learn from the sun alone.

Also, although from groundbased monitoring data we know a lot about magnetic activity in younger solar-like stars (e.g. for one solar-mass stars of Hyades age the rotation period is about a week, the emission from chromospheric plages is some three times greater, the spot area is some 30 times greater, etc.; see Noyes et al 1991), we do not know with confidence for younger solar-like stars how their differential rotation varies with latitude, or anything at all about the variation of their rotation with depth. PRISMA should be able to address such issues, through long term monitoring of selected stars or clusters at the millimagnitude level, and (for fast enough rotators) resolving rotational splitting of $l=1$-modes.

We may hope that PRISMA will be operational long enough to explore many aspects of stellar magnetic activity, manifested through changes of observed parameters as activity changes on individual stars. The finding that solar p-mode frequencies respond to individual episodes of activity rather than simply to the 11-year envelope of activity is encouraging from an observational point of view, for it suggests that an operational life of even a few years may allow PRISMA to obtain important data on analogous frequency changes in other stars. A focussed campaign of PRISMA observations of a few particularly interesting stars should monitor not only changing p-mode frequencies but of course spot modulation (through broadband photometry) and chromospheric and coronal activity (through the ultraviolet spectrometer and XUV telescope). Naturally it should be accompanied by intensive and coordinated groundbased study of time variations in the same stars.

\section{ON SEISMOLOGY OF SUN-LIKE STARS by Timothy M. Brown}

Surely it is reasonable to be optimistic about asteroseismology's favorable impact on stellar evolution theory. The availability of new and precise information about the interior structure of distant Sun-like stars cannot fail to improve our understanding of these objects. Having said this, however, one must add that seismological data, just like other sorts of data, will have their limitations; these should be recognized from the outset.

First, our current understanding suggests that the observable mode frequencies of a star will provide 2 or 3 independent and well-defined numbers about that star. By themselves, these are too few even to define the evolutionary state of the star. Evidently more information will be required just to characterize these stars, let alone to test the physical content of our theories describing them. Second, for the foreseeable future, we will not have oscillations data con- 
cerning a very large number of stars. Whether observed from space or from the ground, the resources demanded by such measurements are simply too great to permit detailed study of more than (say) a few hundred objects.

In view of these facts, it seems clear that for seismological measurements to be worthwhile, they must be able to answer pointed astrophysical questions. This in turn implies that the target list for PRISMA is extraordinarily important. It will be vital to include on the list stars whose special circumstances (as members of star clusters or of binary systems, for example) give us information beyond that available from seismology. Moreover, we must whenever possible use more easily-observed stars ( $\delta$ Scutis, roAp stars) to answer our questions, resorting to Sun-like stars only when necessary. Indeed, my opinion is that PRISMA (and seismology of Sun-like stars generically) can truly succeed only in combination with a vigorous and wide-ranging program of ground-based observations.

\section{WHAT IS NEEDED NEXT? by Françoise Praderie}

This meeting was one with an unusually large audience. The theme "Inside the stars" proved to have a very large federative capacity. It is that same theme which supports the space mission PRISMA. It does it by proposing to complement the theoretical approaches to stellar interiors by the collection of a systematic set of yet unavailable data. PRISMA is a mission dedicated for the stellar community, with a focus directed on the interior of stars. So, to reply to the question: what is needed next?, I will try to show that, from the experimental side, PRISMA is one of the answers.

The mission proposed is a discovery type mission for stellar physics. Not in the sense of unveiling an unknown part of the $\gamma$ ray sky, but in the sense of strengthening the theoretical corpus describing stellar interiors by observational data.

In my view, PRISMA is as fundamental for stellar physics as HIPPARCOS. As some may remember, Hipparcos was for a long time the object of only moderate interest on the part of the stellar community, because the arguments given for the need to go to space were subtle and therefore did not look compelling. PRISMA sometimes suffered from similar reserves, but it seems now that they have been overcome.

Today, and in particular thanks to this meeting, the novelty of going to space to study the interior of stars has become clear. Firstly the Sun opened the paths, with experiments like IPHIR, and soon the instruments on SOHO. Second, the asteroseismology instruments on PRISMA take advantage of using the temporal stability of space, a poorly exploited property, emphasized by $G$. Isaak for quite a long time. Finally $X$ and UV activity tracers will be observed simultaneously on a time scale of order of, or longer than, the rotation period of stars.

Of course the mission will need complementary ground-based observations, such as magnetic field strength in the photosphere, survey of the activity cycle, and photometry of large spots. It will also benefit from basic stellar data as provided by Hipparcos.

But going to space will allow unique performances: for example obtain a high $\mathrm{S} / \mathrm{N}$ ratio in asteroseismology in about one day, or bypass the sensitivity 
of IUE by a fairly considerable factor.

One specific aspect of PRISMA is that three types of instruments will point in the same direction of the sky. Why? To give experimental support to the knowledge of interiors, two approaches will be used: a)the direct sounding of interiors up to the core, $b$ )the indirect record of the magnetic field through effects in the outer layers at various altitudes. Both approaches require long time series with a high duty cycle, and both are strongly related, since the oscillation modes depend on the activity state of the star. The connection between theory and observables is at a different level of achievement in both cases. A lot remains to be done so that activity observables give access to the generation process of the magnetic field. Dynamo theorists will have to guide the PRISMA team, until the reverse situation announced by C. Catala (data to guide the theory) becomes effective.

In conclusion, PRISMA is an opportunity for the stellar physics community. The impact of the stellar physics community on this mission is needed, since it is time to reinforce the scientific objectives, and also to let other communities (planetary, $\mathrm{mm}$, high energies, etc...) know that these objectives are considered of prime value by stellar physicists.

Let us be confident that the general rule will apply: More will be discovered by PRISMA than has been foreseen by its proposers...

\section{CONCLUSIONS by Evry Schatzman}

Two lessons can be drawn from the colloquium. The first one, which is not the least important is a lesson of methodology. The second lesson concerns the future of internal constitution studies. The number of unsolved problems is incredible; from the theoretical point of view, we can show the directions of future work; let's bet that large observational programs will bring unexpected data which will simultaneously help us to understand the inside of the stars and certainly raise new problems.

As far as methodology is concerned, I would like to draw attention to the following points:

- Models, taking into account just one physical process generally do not agree with the observations. This provides evidence for the presence of other physical processes.

- In each phenomenon which we consider are involved a variety of physical processes. It appears necessary now to take into account all physical processes simultaneously. Stars have to be considered globally.

- We should resist to the temptation of using too much phenomenology. Laws of physics are universal, and a law written in a phenomenological form, applying to a particular case, should be checked by applying it to all possible other cases.

I know quite well that such remarks are completely useless. and that any one of us has its own working method. However, I think that future theoretical work should be more rigourous.

It is in such a spirit that we should look at the unsolved problems. Let us consider some examples: 
- The helium content of the Sun. Helioseismology gives $Y=0.23$ in the convective zone. Models give for the inside $\mathrm{Y}=0.27$ or 0.28 . Does gravitational separation explain the difference? And then, what are the constraints on the transport processes? What are the constraints on the hydrodynamics in the radiative zone?

- Abundance of lithium in fast rotating stars. We have the exemple of $\alpha$ Per fast rotators, with a high lithium abundance. Are the present models of lithium burning compatible with these abundances?

- The data on magnetic fields of solar like stars is becoming more and more important. This raises the problem of a better understanding of the origin of stellar magnetic fields and of their evolution.

Finally, let me recall the need of more and more precise observational data. Despite the fact that it does not necessarily involve new spectacular instrumentation, such high quality information is of unesteemable value. Each step of the accuracy performances raises new theoretical problems: this is the way to a better understanding of the inside of stars.

But I would like to end these remarks by a come back to the introduction of Malcolm Longair. The theory of stellar structure gives the key to chronometry of the Universe. At the present epoch, there is a tendency to develop more and more extragalactic research. It is true that this is a field with a large potential number of discoveries. But, as long as galaxies are made of stars, our understanding, our system of datation, our survey of the depths of space depend on our knowledge of stars.

Astrophysics is not made of stellar studies only, but there would not be any astrophysics without it!

\section{REFERENCES}

Biermann, L. 1950, Zeitschrift für Naturforschung A, 56, 65

Charboneau P. and MacGregor K. B., 1992, in: Inside the Stars, eds. W. W. Weiss and $\mathrm{A}$. Baglin, these proceedings

Christensen-Dalsgaard, J., Dilke, F. W. W. and Gough, D. O., 1974, MNRAS 169, 429

Dilke, F. W. W. and Gough, D. O., 1972, Nature 240, 262

Durney, B. R., de Young, D. S. and Passot, T. P., 1990, ApJ 362, 702

Durney, B. R., de Young, D. S. and Roxburgh, I. W., 1992, On the Generation of Large Scale and Turbulent Magnetic Fields in Solar Type Stars, Solar Physics, in press

Faulkner, J., Griffiths, K., and Hoyle, F., 1965. MNRAS 129, 363

Libbrecht, K. G. and Woodard, M. F. 1990, Observations of solar cycle variations in solar p-mode frequencies and splittings. in: Oji Int. Seminar on Progress of Seismology of the Sun and Stars, eds. Y. Osaki and H. Shibahashi, Springer, Berlin 
Noyes, R. W.,Baliunas, S. L, and Guinan, E. F. 1991, What can other stars tell us about the sun?, in: The Solar Interior and Atmosphere, ed A. Cox, W. Livingston, and M. Matthews, U. Ariz. Press, p. 1161

Radick, R. R., Lockwood, G. W., and Baliunas, S. L. 1990, Science 247, 39

Roxburgh, I. W., 1966, MNRAS 132, 201

Roxburgh, I. W., 1976, The Internal Structure of the Sun and Solar Type Stars, in: Basic Mechanisms of Solar Activity, eds. V. Bumba and J. Kleczeck, D. Reidel, Dordrecht, p. 451

Roxburgh, I. W., 1978, $A \& A 65,281$

Roxburgh, I. W., 1984, On Turbulent Mixing, in: Observational Tests of the Stellar Evolution Theory, eds. A. Maeder and A. Renzini, D. Reidel, Dordrecht, p. 519

Roxburgh, I. W., 1985, Solar Physics 100, 21

Roxburgh, I. W., 1987, in: The Internal Solar Angular Velocity, eds. B. R. Durney and S. Sofia, Kluwer, Dordrecht, p. 1

Roxburgh, I. W., 1989, A\&\&A 211, 361

Roxburgh, I. W., 1991, Angular Momentum Transport, Magnetic Fields and Mixing, in: Angular Momentum Evolution of Young Stars, eds. S. Catalano and J. R. Stauffer, Kluwer, Dordrecht, p. 365

Roxburgh, I. W., and Simmons, 1992, Inside the Stars, eds. W. W. Weiss and A. Baglin, these proceedings

Schmidt, J. H. M. M., Rosner, R. and Bohn, H. U., 1984, ApJ 283, 31

Stein, R. and Nordlund, $\AA, 1989$, ApJ 342, L95

Vickers, G., 1970, Problems of Convection in Stars, PhD Thesis, University of London

Woodard, M.F., Kuhn, J.R, Murray, N. and Libbrecht, K.G. 1991, ApJ 373, L81

Zahn, J. P., 1991, $A \& A$ 252, 179 